\title{
An etiological role for aeroallergens and eosinophils in experimental esophagitis
}

\author{
Anil Mishra, Simon P. Hogan, Eric B. Brandt, and Marc E. Rothenberg \\ Division of Pulmonary Medicine, Allergy and Clinical Immunology, Department of Pediatrics, \\ Children's Hospital Medical Center, Cincinnati, Ohio, USA \\ Address correspondence to: Marc E. Rothenberg, Division of Pulmonary Medicine, Allergy and Clinical Immunology, \\ Department of Pediatrics, Children's Hospital Medical Center, Cincinnati, Ohio 45229, USA. \\ Phone: (513) 636-7177; Fax: (513) 636-3310; E-mail: Rothenberg@chmcc.org.
}

Received for publication May 2, 2000, and accepted in revised form November 14, 2000.

Eosinophil infiltration into the esophagus is observed in diverse diseases including gastroesophageal reflux and allergic gastroenteritis, but the processes involved are largely unknown. We now report an original model of experimental esophagitis induced by exposure of mice to respiratory allergen. Allergen-challenged mice develop marked levels of esophageal eosinophils, free eosinophil granules, and epithelial cell hyperplasia, features that mimic the human disorders. Interestingly, exposure of mice to oral or intragastric allergen does not promote eosinophilic esophagitis, indicating that hypersensitivity in the esophagus occurs with simultaneous development of pulmonary inflammation. Furthermore, in the absence of eotaxin, eosinophil recruitment is attenuated, whereas in the absence of IL-5, eosinophil accumulation and epithelial hyperplasia are ablated. These results establish a pathophysiological connection between allergic hypersensitivity responses in the lung and esophagus and demonstrate an etiologic role for inhaled allergens and eosinophils in gastrointestinal inflammation.

J. Clin. Invest. 107:83-90 (2001).

\section{Introduction}

Allergic diseases have reached epidemic proportions in the Western world, inflicting nearly thirty percent of the population (1). Interestingly, this increased prevalence is paralleled by an increase in the severity and spectrum of disorders involving hypersensitivity responses (2). For example, eosinophilia, defined as an accumulation of eosinophils in the blood and/or tissues, is a hallmark feature of allergic diseases and has recently been found to occur in a variety of gastrointestinal disorders $(3,4)$. In disorders such as gastroesophageal reflux, which inflicts nearly $50 \%$ of the population, eosinophil accumulation is observed in the esophagus, suggesting the possibility that allergic hypersensitivity may be contributing to the disease process (5).

Eosinophil infiltration into the esophagus (referred to herein as eosinophilic esophagitis) is a poorly characterized process, frequently occurring in individuals with a variety of diseases including gastroesophageal reflux, allergic gastrointestinal disorders, cow's milk-associated esophagitis, and primary eosinophilic esophagitis syndromes $(2,5,6)$. In patients with primary eosinophilic esophagitis, eosinophils are the predominant infiltrating cell on histological analysis (6-8). The clinical significance of esophageal eosinophils and their role in the pathogenesis of esophageal injury is not known. Although eosinophilia is often associated with allergy, the role of allergy in eosinophilic esophagitis is not known. In some cases, food allergy has been implicated in the pathogenesis of disease, and a food elimination diet or glucocorti- coids ameliorates esophageal eosinophil infiltration and clinical symptoms $(9,10)$. Furthermore, gastroesophageal reflux and esophageal inflammation is more common in patients with asthma, and treatment of these conditions can ameliorate asthma (11). However, it remains unclear whether the pathogenesis of eosinophilic esophagitis is related to the occurrence of atopy and allergen sensitization.

Eosinophil recruitment into inflammatory tissue is a complex process regulated by a number of inflammatory cytokines including IL-3, IL-4, IL-5, GM-CSF, and chemokines like RANTES and eotaxin (12-14). Among the cytokines implicated in modulating eosinophilic inflammation in allergic diseases, only IL-5 and eotaxin selectively regulate eosinophil trafficking. IL-5 regulates growth, differentiation, survival, and activation of eosinophils. Following allergen inhalation, IL-5 has been clearly demonstrated to provide an essential signal for the expansion and mobilization of eosinophils from the bone marrow into the lung (15). In contrast, eotaxin has been shown to have an integral role in regulating the baseline homing of eosinophils into the lamina propria of the gastrointestinal tract at sites distal to the esophagus (e.g., stomach and intestines), but a less significant role in regulating eosinophils in the lung (16-19). In vivo, these two cytokines are likely to cooperate with each other to modulate eosinophil trafficking between the peripheral blood and tissue (20); however, the role of these processes in regulating esophageal eosinophils has not been examined. 
In order to test the hypothesis that eosinophilic esophagitis is mechanistically linked to eosinophilic allergic responses in the lung and to provide insight into the molecular mechanisms that regulate esophageal eosinophils, we have established an experimental model of eosinophilic esophagitis. We have used this experimental regime to dissect the role of the eosinophil-specific cytokines IL-5 and eotaxin in regulating eosinophilic esophagitis. Our results establish that exposure to an inhaled respiratory allergen promotes eosinophilic esophagitis and that common mechanisms regulate eosinophilic inflammation in the respiratory tract and esophagus.

\section{Methods}

Mice. Eight- to ten-week-old BALB/c mice, as well as $\mathrm{C} 57 \mathrm{BL} / 6, \mathrm{FVB} / \mathrm{N}$, and IL-5 gene-targeted mice (C57BL/6), were obtained from The Jackson Laboratory (Bar Harbor, Maine, USA). Eotaxin-deficient inbred mice of the BALB/c background were maintained with age- and sex-matched controls from Taconic Farms (Germantown, New York, USA) as described previously (16). All mice were maintained under specific pathogen-free conditions and according to institutional guidelines.

Histological analysis of eosinophils in the esophagus. The esophagi of adult mice were fixed in 10\% neutral buffered formalin, embedded in paraffin, cut into $5-\mu \mathrm{m}$ sections, fixed to positive charge slides, and immunostained with antiserum against mouse eosinophil major basic protein (anti-MBP) (a kind gift of James and Nancy Lee, Mayo Clinic, Scottsdale, Arizona, USA) as described previously $(17,19)$. In brief, endogenous peroxidase in the tissues was quenched with $0.3 \%$ hydrogen peroxide in methanol followed by nonspecific protein blocking with normal goat serum. Tissue sections were then incubated with rabbit antiMBP $(1: 16,000)$ overnight at $4{ }^{\circ} \mathrm{C}$, followed by $1: 200$ dilution of biotinylated goat anti-rabbit IgG secondary antibody and avidin-peroxidase complex (Vector Laboratories, Burlingame, California, USA) for 30 minutes each. These slides were further developed with nickel diaminobenzidine-cobalt chloride solution to form a black precipitate, and counterstained with nuclear fast red. Replacing the primary antibody with normal rabbit serum ablated the immunostaining as reported (17). Quantification of immunoreactive cells was carried out by counting the positive stained cells under low-power magnification of longitudinal sections using a $10 \times 10 \mu \mathrm{m}$ ocular micrometer (B \& B Microscopes Inc., Warrendale, Pennsylvania, USA), and eosinophil levels are expressed as the number of eosinophils per square millimeter.

Electron microscopic analysis of eosinophils in the esophagus. Esophageal tissue was harvested, cut into 1- to 2$\mathrm{mm}$ cubes, and fixed in $3 \%$ glutaraldehyde in $0.175 \mathrm{M}$ Cacodylate buffer, $\mathrm{pH} 7.4$, overnight at $4^{\circ} \mathrm{C}$. Tissue was then post-fixed in $1 \%$ osmic acid in Cacodylate buffer for 1 hour and dehydrated in ethanol. Follow- ing dehydration, the tissue was saturated in propylene oxide for two changes followed by embedding in LX 112 resin (Ladd Research Industries, Burlington, Vermont, USA) and polymerized for 2 days at $60^{\circ} \mathrm{C}$. Sections were cut, mounted on copper grids, stained with uranyl acetate and lead citrate, and examined with a Zeiss EM 912 electron microscope (Carl Zeiss Inc., Thornwood, New York, USA).

Allergen treatment of mice. A mouse model of allergic lung disease was established using methods described previously $(19,21)$. In brief, mice were lightly anesthetized with Metofane inhalation (methoxy-fluorane; Schering-Plough Animal Health, Union, New Jersey, USA), and $100 \mu \mathrm{g}(50 \mu \mathrm{l})$ of Aspergillus fumigatus (Bayer Corp., Spokane, Washington, USA), $60 \mu \mathrm{g}$ (50 $\mu \mathrm{l})$ of dust mite mixture (Bayer Corp.), or $50 \mu \mathrm{l}$ of normal saline alone was applied to the nasal or oral cavity using a micropipette with the mouse held in the supine position. After instillation, mice were held upright until alert. In other experiments, $100 \mu \mathrm{l}(100$ $\mu \mathrm{g}$ ) of $A$. fumigatus was applied to the stomach using an 18-gauge blunt-ended stainless steel animal feeding needle (Popper and Sons Inc., New Hyde Park, New York, USA). After three treatments per week for 3 weeks, mice were sacrificed between 18 and 20 hours after the last intranasal challenge. In some experiments, mice were pretreated by intraperitoneal injection of $0.5,0.75$, and $1.0 \mathrm{mg}$ of anti-mouse IL-5 (TRFK-5) on the first day of the three weekly allergen treatments, respectively (22). Controls were also first treated with the same dose of antibodies before challenging with normal saline. The TRFK-5 hybridoma was purchased from American Type Culture Collection (Rockville, Maryland, USA), grown in ascites fluid, and the antibody was partially purified by ammonium sulfate precipitation. In some experiments, anesthetized mice were exposed to nine doses $(25 \mu \mathrm{l})$ of intranasal Ascaris pseudocoelomic fluid (APF; a kind gift of Joseph Urban, US Department of Agriculture, Bellville, Maryland, USA, and Fred Finkelman, University of Cincinnati, Cincinnati, Ohio, USA) (23-26) on alternate days and analyzed 18 hours after the last allergen challenge. In other experiments, mice were sensitized by intraperitoneal injection of $50 \mu \mathrm{g}$ of ovalbumin (OVA) and $1 \mathrm{mg}$ alum in $0.9 \%$ sterile saline on two occasions separated by 14 days. On day 15 , mice were subsequently anesthetized and intranasally exposed to $150 \mu \mathrm{g}$ of OVA $(50 \mu \mathrm{l})$ on seven occasions over 10 days, followed by analysis 18 hours after the last dose.

Analysis of epithelial cell proliferation. In order to determine the degree of epithelial cell proliferation, $5^{\prime}$-bromodeoxyuridine (5'-BrdU) (Zymed Laboratories Inc., South San Francisco, California, USA) incorporation analysis was performed according to previously reported methods (27). In brief, saline or Aspergillustreated mice were injected intraperitoneally with 0.25 $\mathrm{ml}$ of $5^{\prime}$-BrdU, 3 hours before sacrifice. The esophagus was fixed with $10 \%$ neutral buffered formalin (Sigma 


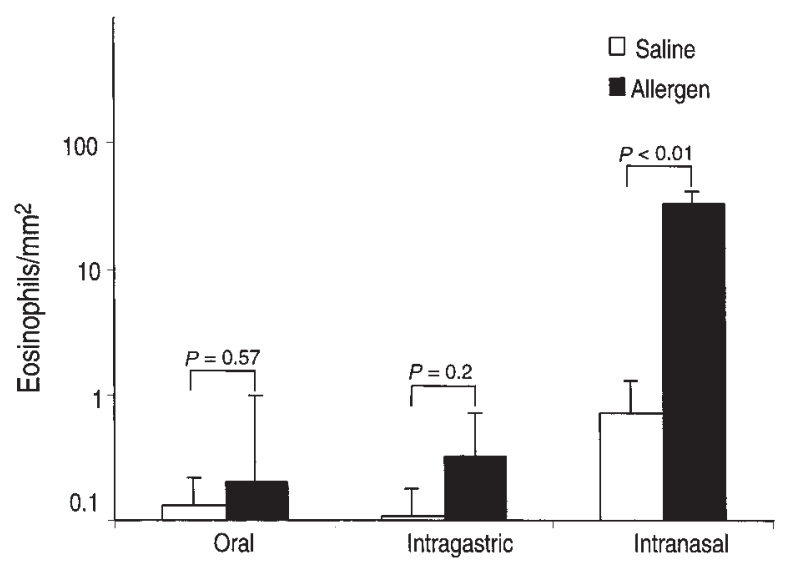

Figure 1

The effect of oral, intragastric, and intranasal allergen challenge on eosinophil levels in the esophagus. Mice (BALB/c) were challenged with repeated inoculations of oral, intragastric and intranasal allergen. Eighteen hours after the last allergen or placebo challenge, the mice were sacrificed and eosinophil levels were evaluated in the esophagus. Data are expressed as mean \pm SEM; $n=8-9$ mice.

Chemical Co., St. Louis, Missouri, USA) for 24 hours. After fixation, the tissue was embedded in paraffin and $5-\mu \mathrm{m}$ sections were processed using standard histological approaches. Tissues were digested with trypsin $(0.125 \%)$ for 3 minutes at $37^{\circ} \mathrm{C}$ followed by incubation for 30 minutes at room temperature. Sections were washed with PBS three times for 2 minutes and further incubated with monoclonal biotinylated anti-BrdU antibody (Zymed Laboratories Inc.) for 60 minutes at room temperature. Negative controls included replacing the primary antibody with PBS, and positive controls were provided by the manufacturer. BrdU nuclear incorporated positive cells were detected with streptavidin-peroxidase and diaminobenzidine substrate (Zymed Laboratories Inc.) followed by counterstaining with hematoxylin.

Broncho-alveolar lavage fluid analysis. Allergen- or saline-challenged mice were sacrificed, the trachea was cannulated after midline neck incision, and the lungs were lavaged twice with $0.8 \mathrm{ml}$ normal saline containing $0.5 \mathrm{mM}$ EDTA. The recovered broncho-alveolar lavage fluid (BALF) was centrifuged at $400 \mathrm{~g}$ for 10 minutes at $4{ }^{\circ} \mathrm{C}$, and cells were counted with a hematocytometer. Cytospin preparations were stained with Diff-Quick (Dade Diagnostics of Puerto Rico Inc., Aguada, Puerto Rico).

Blood eosinophil analysis. Peripheral blood samples were collected in heparinized tubes (Becton Dickinson and Co., Franklin Lakes, New Jersey, USA) by tail bleeding. Blood eosinophil levels were determined by counting cells with a Neubauer hemacytometer (Fisher Scientific Co., Pittsburgh, Pennsylvania, USA) and by staining whole blood with Discombe's solution (28).

Statistical analysis. Data are expressed as mean \pm SEM. Statistical significance comparing different sets of mice was determined by Student's $t$ test.

\section{Results}

Intranasal allergen induces eosinophilic esophagitis. We have recently reported that eosinophils are readily detectable in the lamina propria of gastrointestinal segments from the stomach to the colon (19). In contrast to these gastrointestinal segments, the esophagus has barely detectable eosinophils. For example, quantification of eosinophils by anti-MBP immunohistological staining revealed that the stomach and small intestine have $36.9 \pm 11.9$ and $54.9 \pm 17.4$ eosinophils per square millimeter in the lamina propria, respectively. In contrast, the esophagus has $0.2 \pm 0.09$ eosinophils per square millimeter (mean \pm SEM, $n=10-12$ ). Since eosinophils accumulate in the esophagi of individuals with a variety of disorders, we were interested in developing an experimental system for inducing esophageal eosinophilia. We hypothesized that eosinophil recruitment to the esophagus might be induced by exposure to mucosal allergens, since eosinophil tissue accumulation is associated with allergic disorders. We therefore exposed mice to repeated inoculations of $A$. fumigatus antigens by oral, intragastric, and intranasal approaches and analyzed the level of eosinophils in the esophagus by anti-MBP
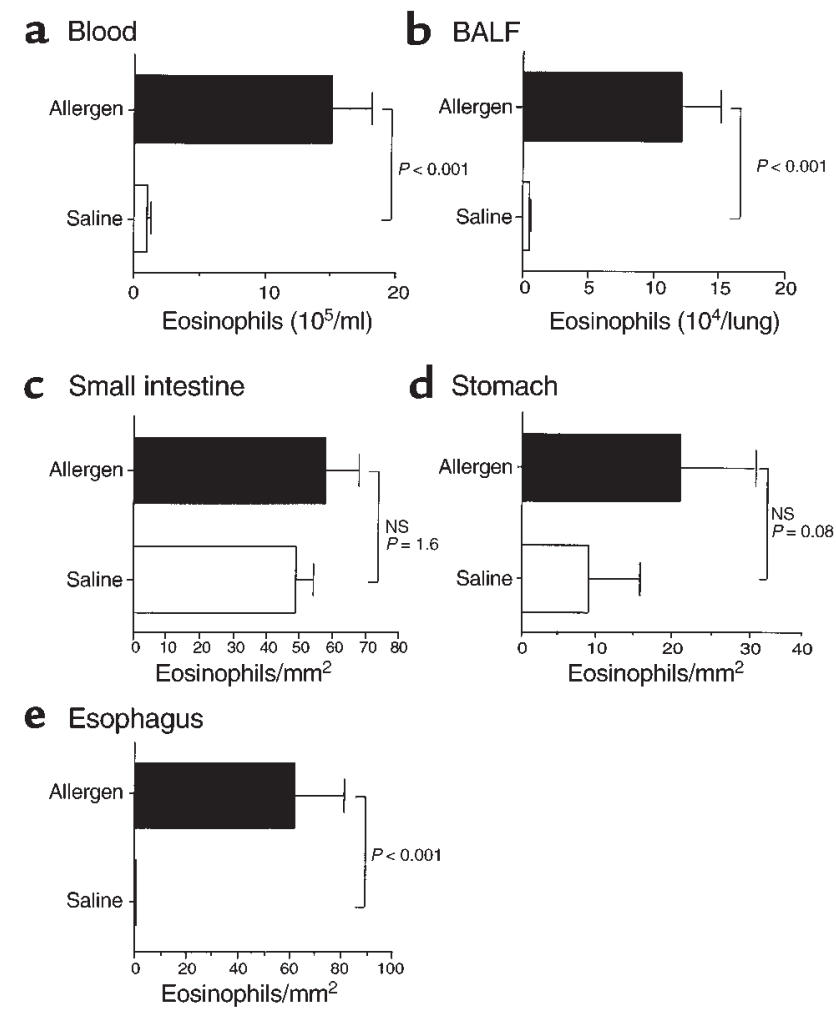

Figure 2

The effect of intranasal allergen challenge on eosinophil levels. Mice were challenged with allergen or saline intranasally, and 18 hours after the last allergen exposure the mice were sacrificed and eosinophil levels in the blood (a), BALF (b), small intestine (c), stomach (d), and esophagus (e) were determined. Data are expressed as mean $\pm \operatorname{SEM}(n=8-12$ mice in each group). The mouse strains were a mixture of $129 \mathrm{SvEv}, \mathrm{BALB} / \mathrm{c}$, and FVB/N. NS, not significant. 
immunostaining (Figure 1). Exposure of mice to oral or intragastric allergen failed to elicit eosinophilia; in contrast, mice exposed to intranasal allergen developed pronounced esophageal eosinophilia (Figure 1). Eosinophils in the esophagi of Aspergillus-treated mice were $34 \pm 6$ eosinophils per square millimeter and remained low in saline-treated mice.

Eosinophilic esophagitis is associated with airway inflammation. We were next interested in establishing whether eosinophilic esophagitis and airway inflammation were specifically linked. We first determined the tissue distribution of eosinophilic inflammation following intranasal allergen by examining eosinophils in the blood, BALF, stomach, and small intestine. Allergen challenge induced a significant increase in the level of eosinophils in the blood, BALF, and esophagus (Figure 2). In contrast to the esophagus, eosinophil levels in the stomach and small intestine did not significantly increase following allergen challenge. Similarly, exposure of mice to oral or intragastric allergen did not induce eosinophilia in the blood, BALF, stomach, and small intestine (data not shown). As a control, the number of eosinophils in the heart, an organ without resident eosinophils at base line, was shown not to increase following intranasal allergen challenge (data not shown). In order to rule out the possibility that allergen sensitization was dependent upon the nasal mucosa rather than the lungs, we next determined whether intranasal exposure to A. fumigatus antigen (in the absence of pulmonary exposure) was sufficient for the induction of eosinophilic esophagitis. To accomplish this, we exposed anesthetized and nonanesthetized mice to nine intranasal allergen inoculations; however, only the anesthetized mice developed pulmonary and esophageal eosinophilic inflammation (data not shown). Collectively, these results suggest that induction of eosinophilic esophagitis occurs as a result of allergen sensitization in the respiratory tract followed by topical delivery of allergen to the esophagus. In support of this, we have found that about $33 \%$ of the intranasal inoculation to anesthetized mice was deposited in the gastrointestinal tract, consistent with the previous literature (29).

In order to determine whether other antigens were able to elicit esophageal inflammation, we induced allergic airway inflammation with two other allergens, APF and OVA, two potent inducers of experimental eosinophilic lung inflammation (23-26, 30, 31). Mice exposed to nine doses of intranasal APF developed significant esophageal eosinophilia compared with saline exposed controls $(12.1 \pm 7.6$ and $0.44 \pm 0.9$ eosinophils $/ \mathrm{mm}^{2}$ for APF and saline exposed mice, respectively; $P<0.02$ ). Exposure of OVA-sensitized mice to repeated doses of intranasal OVA also induced esophageal eosinophilia $(6.4 \pm 2.2$ and $0.72 \pm 0.7$ eosinophils $/ \mathrm{mm}^{2}$ for OVA and saline exposed mice, respectively; $P<0.05)$. Taken together, these results support an etiologic association between allergic eosinophilic responses in the lung and esophagus and suggest a relatively dominant role for A. fumigatus in initiating this process.

Pathological changes associated with experimental eosinophilic esophagitis. Histological examination of the esophagi of untreated and saline-treated control mice revealed the presence of very few eosinophils in the tissue. No eosinophils were detected in most fields, as shown in Figure 3a. Allergen-induced eosinophil accumulation occurred in all regions of the esophagus,

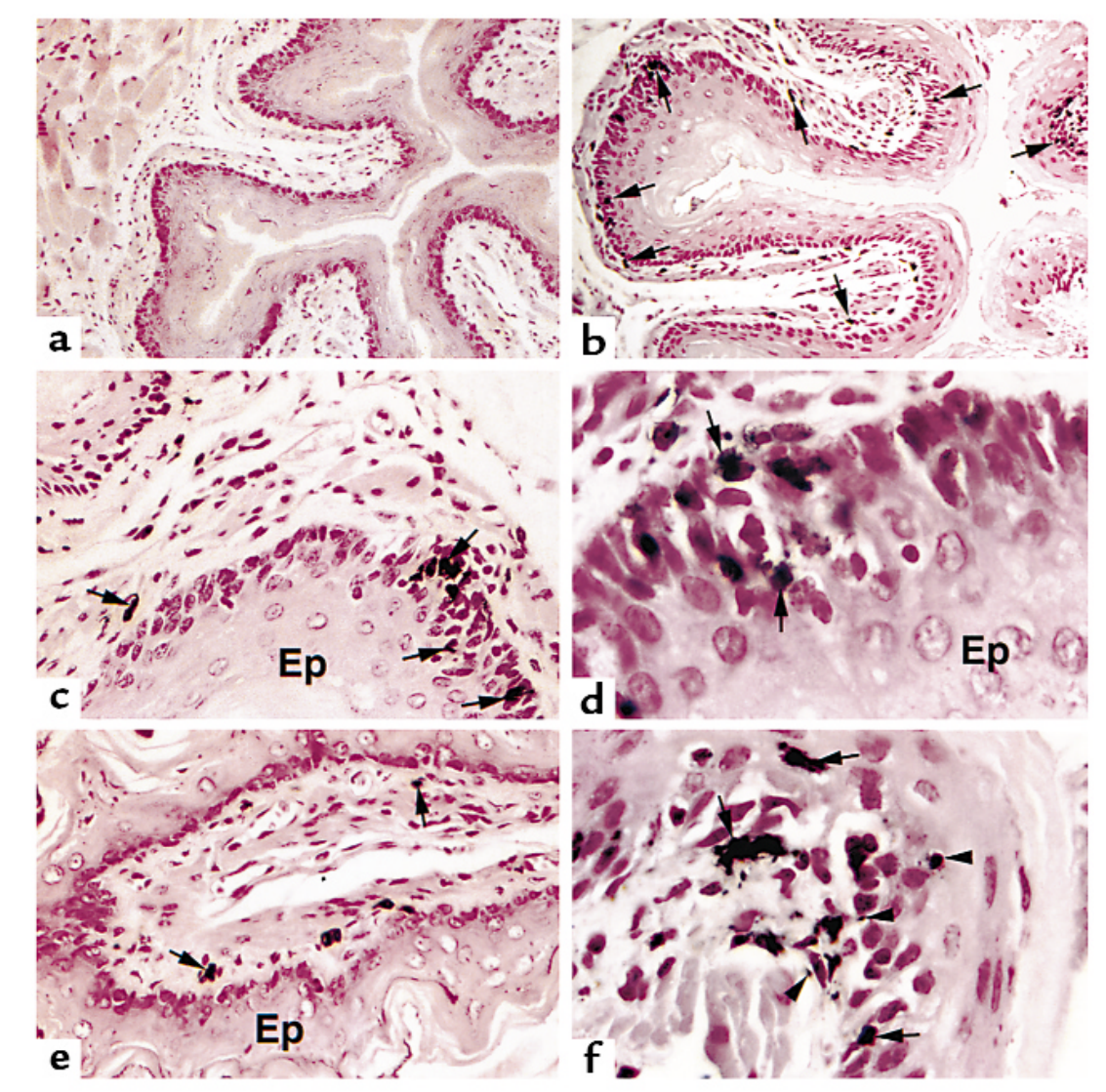




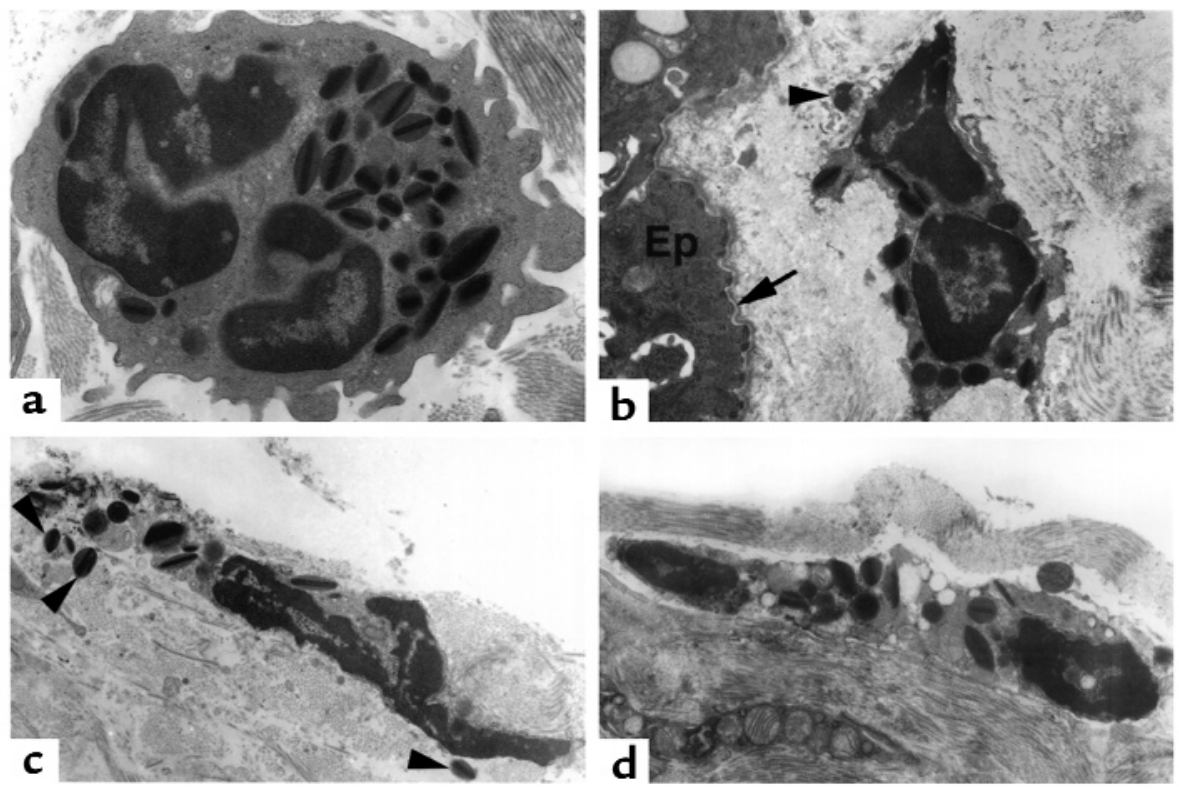

\section{Figure 4}

Electron microscopic analysis of esophageal eosinophils. Mice (BALB/c) were treated with saline (a) or allergen (b-d), and electron microscopy was performed on the esophagus. Representative electron micrographs are shown. (a) A healthy-appearing eosinophil with an intact plasma membrane, a segmented nucleus, and abundant electron-dense granules with characteristic cores and matrices. (b-d) Eosinophil plasma membranes are disrupted, the nuclei are undergoing chromolysis, and there are readily detectable extracellular eosinophil granules (shown with arrowheads). The location of an eosinophil next to the epithelial layer (Ep) and below the basement membrane (arrow) is illustrated in b. Original magnifications are $\times 8000(\mathbf{a}), \times 5000(\mathbf{b}$ and $\mathbf{d})$, and $\times 6300(\mathbf{d})$.

including the epithelial layer and muscularis propria; however, the eosinophils were predominantly localized to the lamina propria and submucosa regions (Figure 3, b-f). Eosinophils were frequently found at the junction of the lamina propria and the basal layer of the epithelium (Figure 3, b-f). In addition, eosinophils were found to penetrate the epithelial layer and were associated with epithelial cell dysplasia, noted by the loss of normal orientation of one epithelial cell to another (Figure 3, d and f). Interestingly, MBP immunoreactivity was detected in extracellular areas, suggesting release of eosinophil products into the interstitium (Figure $3, \mathrm{~d}$ and $\mathrm{f}$ ).

In order to analyze eosinophil morphology further, an electron microscopic analysis of the esophageal tissue was conducted. Esophageal eosinophils from allergen-challenged mice were primarily found in the connective tissue of the submucosa and lamina propria in association with collagen matrices. Approximately half of the eosinophils had features characteristic of healthy eosinophils including intact plasma membranes, heterochromatic segmented nuclei, and abundant granules with characteristic electron dense cores and matrices (Figure 4a) (32). However, half of the eosinophils appeared to be undergoing cell death, as indicated by the disruption of their plasma membranes, by the occurrence of nuclear chromatolysis, and by the detection of eosinophil granules in the extracellular spaces adjacent to these eosinophils (Figure 4, b-d). Interestingly, some of these granules had a preserved core structure, suggesting that they were released as intact granules. These ultrastructural features, mainly eosinophil cytolysis and the release of free proteinladen eosinophil granules, are the most common finding associated with eosinophilic mucosal inflammation in human disorders $(32,33)$.

Because eosinophil accumulation in the epithelial layer was associated with epithelial changes, we hypothesized that allergen challenge might be inducing epithelial cell hyperplasia, a finding frequently seen in human esophagitis $(34,35)$. In order to test this, we measured the incorporation of BrdU after its administration to mice following the last allergen challenge. This analysis revealed that allergen-challenged mice had an approxi-

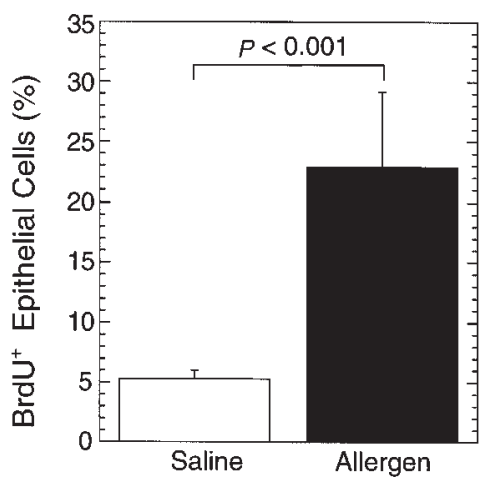

\section{Figure 5}

Epithelial proliferation following allergen challenge. Mice (BALB/c) were challenged with saline or allergen intranasally and the incorporation of $\mathrm{BrdU}$ in the epithelial layer was measured 3 hours after the last challenge. The results are mean $\pm \operatorname{SEM}(n=4-5$ mice $)$. 

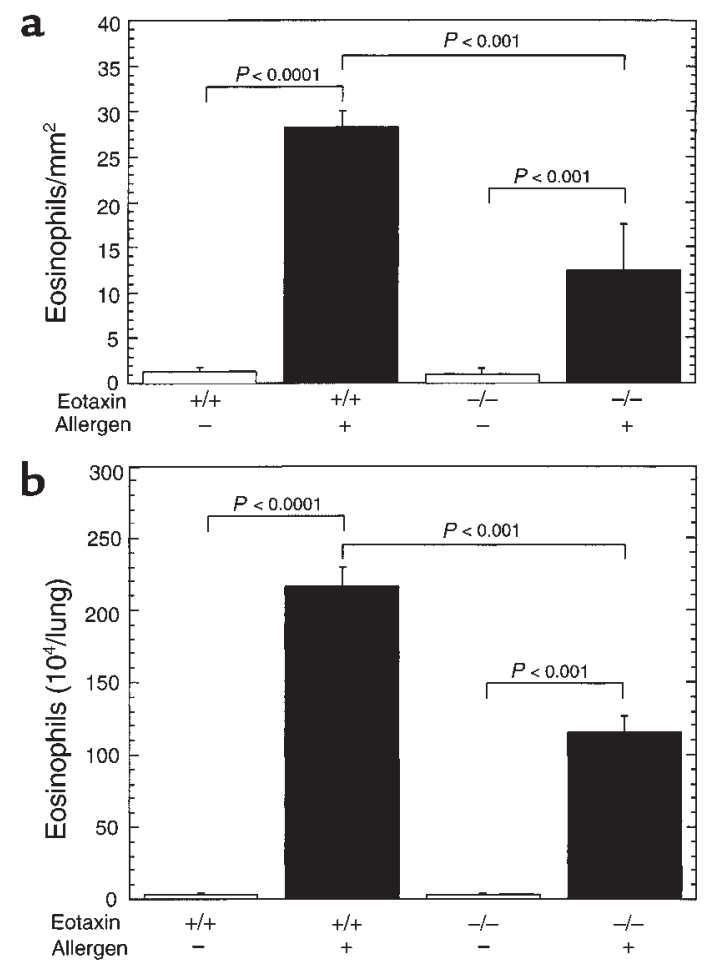

Figure 6

Induction of eosinophilic esophagitis in eotaxin gene-targeted mice. Wild-type $(+/+)$ or eotaxin-deficient (-/-) mice (BALB/c) were challenged with control saline $(-)$ or allergen $(+)$, and the number of eosinophils in the esophagus (a) was determined by anti-MBP staining and in the lung fluid (b) by differential counting of cells in BALF. The results are expressed as mean $\pm \operatorname{SEM}(n=4-6$ mice).

mately fivefold increase in BrdU incorporation compared with mice that were challenged with saline (Figure 5). These results establish that intranasal allergen challenge induces an experimental model of esophagitis characterized by eosinophil accumulation, free eosinophil granules, and epithelial hyperplasia.

Eotaxin is partially required for allergen-induced experimental eosinophilic esophagitis. We were next interested in establishing whether eotaxin had an essential role in promoting esophageal eosinophils following allergen challenge. We addressed this by inducing experimental eosinophilic esophagitis in eotaxin gene-targeted mice. The level of eosinophils in the esophagus was markedly increased in allergen-challenged wild-type mice compared with eotaxin-deficient mice (Figure 6a). In the absence of the eotaxin, there was a twofold reduction in the number of eosinophils in the esophagus compared with allergen-challenged wild-type mice. However, in the absence of eotaxin, allergen challenge still induced about tenfold more eosinophils compared with placebo treatment. The partial role for eotaxin in regulating esophageal eosinophilia is similar to its partial role in the lung as assessed by allergen-induced eosinophil accumulation in the BALF (Figure 6b).

$I L-5$ is required for allergen-induced experimental eosinophilic esophagitis. We were next interested in estab- lishing whether IL-5 had an essential role in promoting esophageal eosinophilia following allergen challenge. We addressed this by inducing experimental eosinophilic esophagitis in wild-type and IL-5 genetargeted mice (Figure 7). Exposure of IL-5 gene-targeted mice to intranasal allergen did not induce eosinophilic esophagitis, while wild-type control mice developed marked eosinophilic esophagitis (Figure 7a). An independent method was also used to confirm the critical role of IL-5 in eosinophilic esophagitis by treatment of mice with neutralizing anti-IL-5 serum. Anti-IL-5 allergen-treated mice had markedly reduced esophageal eosinophils compared with control allergen-treated mice ( $4.6 \pm 0.9$ vs. $28.6 \pm 6.9$ [mean \pm SEM, $n=4]$ for anti-IL-5 and control mice, respectively).

We were next interested in determining whether the lack of allergen-induced esophageal eosinophils in IL5 -deficient mice was associated with protection from allergen-induced epithelial cell hyperplasia. We therefore measured the incorporation of BrdU after its administration to wild-type and IL-5-deficient mice
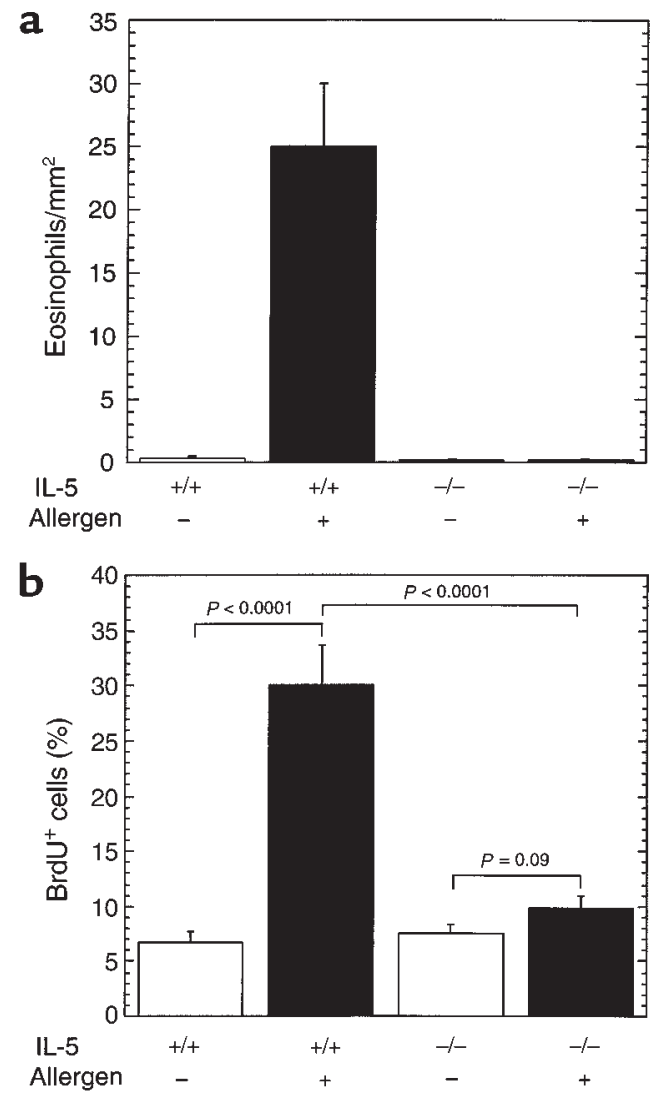

\section{Figure 7}

The role of IL-5 in allergen-induced eosinophil recruitment and epithelial hyperplasia in the esophagus. Wild-type $(+/+)$ and IL-5 gene-targeted (-/-) mice $(\mathrm{C} 57 \mathrm{BL} / 6)$ were subjected to the allergen challenge protocol by treatment with control saline (-) or allergen $(+)$ inoculations. (a) The number of eosinophils in the esophagus was determined by anti-MBP staining. (b) The incorporation of BrdU in the epithelial layer was measured 3 hours after the last challenge. The results are mean $\pm \operatorname{SEM}(n=4-5$ mice $)$. 
following the last allergen challenge. This analysis revealed that allergen-induced epithelial hyperplasia was ablated in IL-5-deficient mice (Figure 7b). These data suggested that eosinophils were causally related to the pathological features of experimental esophagitis.

\section{Discussion}

Eosinophil infiltration into the esophagus is a commonly observed medical problem in patients with diverse diseases including gastroesophageal reflux, drug reactions, allergic eosinophilic esophagitis, eosinophilic gastroenteritis, and primary eosinophilic esophagitis $(2,3,7,34,35)$. Recent clinical studies have suggested that the level of eosinophils in the esophagus negatively correlates with response to conventional gastroesophageal reflux therapy (36). Additionally, the clinical symptoms and the eosinophilic infiltrations can be ameliorated by a food allergen elimination diet or anti-inflammatory therapy (cromoglycate or glucocorticoids) in some patients $(9,10)$. This suggests that sensitized individuals may develop esophageal eosinophilic inflammation in response to exposure to food allergens. However, the role of allergens in the induction of eosinophilia in the esophagus has been debated, since there is no direct causal evidence proving this linkage (6). We now demonstrate that exposure of anesthetized mice to repeated challenges of aeroallergens using a standard protocol to induce allergic airway inflammation promotes marked eosinophilic esophagitis. The accumulation of eosinophils, especially intraepithelial eosinophils, the detection of extracellular granules, and epithelial cell hyperplasia mimic the pathophysiological changes observed in individuals with various forms of eosinophilic esophagitis (6). Of note, the detection of protein-laden free eosinophil granules is a common pathological observation in human eosinophilic inflammation (33). It is likely that sensitization occurs by pulmonary exposure, since oral or intragastric allergen exposure alone fails to elicit eosinophilia. In support of this, intranasal inoculation to nonanesthetized mice, which bypasses allergen delivery to the lung, failed to induce pulmonary and esophageal eosinophilic inflammation. Mechanistically, it is interesting to speculate that sensitization occurs through the respiratory tract (lung), and when the sensitized mice are subsequently exposed to the oral allergens deposited by the intranasal application, they develop a hypersensitivity response that leads to eosinophil infiltration into the esophagus. Taken together, this study suggests that aeroallergens may contribute to the development of eosinophilic esophagitis in sensitized atopic patients.

In this study we also demonstrate that IL-5 has a central role in regulating eosinophil accumulation in the esophagus. In the absence of IL-5 (by analysis of genetargeted mice or anti-IL-5-treated mice), esophageal eosinophilia is markedly reduced. Thus, the esophagus likely uses similar pathways, involving $\mathrm{CD} 4^{+} \mathrm{T}$ cells and
IL-5, for the induction of eosinophilic inflammation as those operational during allergic pulmonary inflammation. The lack of allergen-induced epithelial hyperplasia in IL-5-deficient mice suggests an etiological role for eosinophils in promoting epithelial proliferation. Eosinophils are sources of a variety of cytokines, including growth factors (e.g., TGF- $\alpha$ and $-\beta$ ), that may be involved in promoting epithelial hyperplasia (12). In addition to regulating eosinophilopoiesis, IL-5 primes eosinophils to have enhanced responsiveness to chemokines such as eotaxin (37). We have also demonstrated that, following allergen challenge, the accumulation of eosinophils in the esophagus is dependent in part upon eotaxin. However, eotaxin-deficient mice still show markedly elevated eosinophil levels in the esophagus compared with base-line wild-type mice or mice treated with placebo alone. This indicates the cooperation of eotaxin with other eosinophil-active chemoattractants in the regulation of eosinophil trafficking to the esophagus. Consistent with this, eosinophils respond to a variety of chemokines including other CCR3 ligands (monocyte chemoattractant protein-2 [MCP-2] and MCP-3, RANTES, and eotaxin-2 and -3) (12-14). Thus, the requirements for IL-5 and eotaxin in regulating eosinophils in the esophagus are similar to their respective roles in the lungs.

In summary, these investigations dissect the cellular and molecular mechanisms involved in eosinophil homing into the esophagus. We demonstrate that intranasal allergen exposure induces marked eosinophil infiltration into the esophagus. This suggests that eosinophilic esophagitis can be mediated by extrinsic allergens and establishes a causal link between the development of allergic hypersensitivity in the respiratory tract and in the esophagus, implicating an etiologic role for aeroallergens in the pathogenesis of esophagitis. We propose that aeroallergens may be contributing to the pathogenesis of esophageal inflammation in a subset of patients with primary eosinophilic esophagitis and gastroesophageal reflux disorders. These results suggest that a subgroup of patients with eosinophilic esophagitis may benefit from control of aeroallergen sensitization and/or exposure. Furthermore, the demonstration that IL-5, which is required for pulmonary eosinophilia, is also required for eosinophil infiltration in the esophagus further substantiates immunological cooperativity between the immune responses in the lung and esophagus. It has long been debated whether eosinophils have a pathological role in gastrointestinal inflammatory disorders. The demonstration that allergen-induced epithelial hyperplasia is ablated in IL-5 gene-targeted mice indicates that eosinophils are likely to be causally related to gastrointestinal pathology. These data suggest that anti-IL-5 therapy, which is currently being tested for the treatment of asthma, may be a rational approach for the treatment of eosinophilic esophageal disorders. It is hoped that this novel experimental regime 
for the induction of eosinophilic esophagitis will facilitate future investigations designed to understand the pathophysiology and treatment of inflammatory disorders of the esophagus.

\section{Acknowledgments}

The authors would like to thank Ann Dvorak for expert review of the electron microscopy; Mitchell Cohen, Susan Wert, Nives Zimmermann, David Witte, Fred Finkelman, and Sam Pope for helpful discussions and review of the manuscript; Alicia Emly for graphic assistance; and Irene Hofman for technical assistance with electron microscopy. This work was supported in part by the National Health Medical Research Council (Canberra, Australia) C.J. Martin Post-doctoral Fellowship (to S.P. Hogan), NIH grants R01 AI42242-02 and R01 AI45898-01 (both to M.E. Rothenberg), and the Human Frontier Science Program (to M.E. Rothenberg).

1. Holgate, S.T. 1999. The epidemic of allergy and asthma. Nature. 402:B2-B4.

2. Sampson, H.A. 1999. Food allergy. Part 1: immunopathogenesis and clinical disorders. J. Allergy Clin. Immunol. 103:717-728.

3. Furuta, G.T., Ackerman, S.J., and Wershil, B.K. 1995. The role of the eosinophil in gastrointestinal diseases. Curr. Opin. Gastroenterol. 11:541-547.

4. Burks, A.W. 1998. The spectrum of food hypersensitivity: where does it end? J. Pediatr. 133:175-176.

5. Winter, H.S., et al. 1982. Intraepithelial eosinophils: a new diagnostic criterion for reflux esophagitis. Gastroenterology. 83:818-823.

6. Furuta, G.T. 1998. Eosinophils in the esophagus: acid is not the only cause. J. Pediatr. Gastroenterol. Nutr. 26:468-471.

7. Walsh, S.V., et al. 1999. Allergic esophagitis in children: a clinicopathological entity. Am. J. Surg. Pathol. 23:390-396.

8. Orenstein, S.R., et al. 2000. The spectrum of pediatric eosinophilic esophagitis beyond infancy: a clinical series of 30 children. Am. J. Gastroenterol. 95:1422-1430.

9. Kelly, K.J., et al. 1995. Eosinophilic esophagitis attributed to gastroesophageal reflux: improvement with an amino acid-based formula. Gastroenterology. 109:1503-1512.

10. Liacouras, C.A., Wenner, W.J., Brown, K., and Ruchelli, E. 1998. Primary eosinophilic esophagitis in children: successful treatment with oral corticosteroids. J. Pediatr. Gastroenterol. Nutr. 26:380-385.

11. Harding, S.M. 1999. Gastroesophageal reflux and asthma: insight into the association. J. Allergy Clin. Immunol. 104:251-259.

12. Rothenberg, M.E. 1998. Eosinophilia. N. Engl. J. Med. 338:1592-1600.

13. Nickel, R., Beck, L.A., Stellato, C., and Schleimer, R.P. 1999. Chemokines and allergic disease. J. Allergy Clin. Immunol. 104:723-742.

14. Lukacs, N.W., Oliveira, S.H., and Hogaboam, C.M. 1999. Chemokines and asthma: redundancy of function or a coordinated effort? J. Clin. Invest. 104:995-999.

15. Foster, P.S., Hogan, S.P., Ramsay, A.J., Matthaei, K.I., and Young, I.G. 1996. Interleukin 5 deficiency abolishes eosinophilia, airways hyperreactivity, and lung damage in a mouse asthma model. J. Exp. Med. 183:195-201.

16. Rothenberg, M.E., MacLean, J.A., Pearlman, E., Luster, A.D., and Leder, P. 1997. Targeted disruption of the chemokine eotaxin partially reduces antigen-induced tissue eosinophilia. J. Exp. Med. 185:785-790.

17. Matthews, A.N., et al. 1998. Eotaxin is required for the baseline level of tissue eosinophils. Proc. Natl. Acad. Sci. USA. 95:6273-6278.

18. Yang, Y., Loy, J., Ryseck, R.P., Carrasco, D., and Bravo, R. 1998. Antigeninduced eosinophilic lung inflammation develops in mice deficient in chemokine eotaxin. Blood. 92:3912-3923.

19. Mishra, A., Hogan, S.P., Lee, J.J., Foster, P.S., and Rothenberg, M.E. 1999. Fundamental signals regulate eosinophil homing to the gastrointestinal tract. J. Clin. Invest. 103:1719-1727.

20. Collins, P.D., Marleau, S., Griffiths-Johnson, D.A., Jose, P.J., and Williams, T.J. 1995. Cooperation between interleukin-5 and the chemokine eotaxin to induce eosinophil accumulation in vivo. J. Exp. Med. 182:1169-1174.

21. Huang, W.W., et al. 1998. Molecular and biological characterization of the murine leukotriene $\mathrm{B}_{4}$ receptor expressed on eosinophils. J. Exp. Med. 188:1063-1074.

22. Schumacher, J.H., et al. 1988. The characterization of four monoclonal antibodies specific for mouse IL-5 and development of mouse and human IL-5 enzyme-linked immunosorbent. J. Immunol. 141:1576-1581.

23. Marretta, J., and Casey, F.B. 1979. Effect of Ascaris suum and other adjuvants on the potentiation of the IgE response in guinea-pigs. Immunology. 37:609-613.

24. Greenspon, L.W., White, J., Shields, R.L., Fugner, A., and Gold, W.M. 1986. Purification of Ascaris suum antigen: its allergenic activity in vitro and in vivo. J. Allergy Clin. Immunol. 77:443-451.

25. Hamel, R., McFarlane, C.S., and Ford-Hutchinson, A.W. 1986. Late pulmonary responses induced by Ascaris allergen in conscious squirrel monkeys. J. Appl. Physiol. 61:2081-2087.

26. Xia, Y., et al. 2000. The ABA-1 allergen of Ascaris lumbricoides: sequence polymorphism, stage and tissue-specific expression, lipid binding function, and protein biophysical properties. Parasitology. 120:211-224

27. Donaldson, K., Brown, D.M., Miller, B.G., and Brody, A.R. 1995. Bromo-deoxyuridine (BRDU) uptake in the lungs of rats inhaling amosite asbestos or vitreous fibres at equal airborne fibre concentrations. Exp. Toxicol. Pathol. 47:207-211.

28. Discombe, G. 1946. Criteria of eosinophilia. Lancet. 1:195.

29. Wills-Karp, M. 1999. Allergen-induced airway inflammation in mice. In In vivo models of inflammation. D.W. Morgan and L.A. Marshall, editors. Birkhäuser Publishing Ltd. Basel, Switzerland. 137-158.

30. Renz, H., et al. 1992. Aerosolized antigen exposure without adjuvant causes increased IgE production and increased airway responsiveness in the mouse. J. Allergy Clin. Immunol. 89:1127-1138.

31. Wills-Karp, M. 1999. Immunologic basis of antigen-induced airway hyperresponsiveness. Annu. Rev. Immunol. 17:255-281.

32. Dvorak, A.M., and Ishizaka, T. 1994. Human eosinophils in vitro. An ultrastructural morphology primer. Histol. Histopathol. 9:339-374.

33. Erjefalt, J.S., et al. 1999. Allergen-induced eosinophil cytolysis is a primary mechanism for granule protein release in human upper airways. Am. J. Respir. Crit. Care Med. 160:304-312.

34. Kelly, K.J. 2000. Eosinophilic gastroenteritis. J. Pediatr. Gastroenterol. Nutr. 30(Suppl.):S28-S35.

35. Sampson, H.A., and Anderson, J.A. 2000. Summary and recommendations: classification of gastrointestinal manifestations due to immunologic reactions to foods in infants and young children. J. Pediatr. Gastroenterol. Nutr. 30(Suppl.):S87-S94.

36. Ruchelli, E., Wenner, W., Voytek, T., Brown, K., and Liacouras, C. 1999. Severity of esophageal eosinophilia predicts response to conventional gastroesophageal reflux therapy. Pediatr. Dev. Pathol. 2:15-18.

37. Lamkhioued, B., et al. 1997. Increased expression of eotaxin in bronchoalveolar lavage and airways of asthmatics contributes to the chemotaxis of eosinophils to the site of inflammation. J. Immunol. 159:4593-4601 